\title{
CORRELATED DEFAULT WITH INCOMPLETE INFORMATION
}

\author{
Kay Giesecke* \\ Humboldt-Universität zu Berlin
}

November 1, 2000; this version December 12, 2001

\begin{abstract}
We propose a model of correlated multi-firm default with incomplete information. While public bond investors observe issuers' assets and defaults, we suppose that they are not informed about the threshold asset level at which a firm is liquidated. Bond investors form instead a prior on these thresholds. Stochastic dependence between default events is induced through correlated asset values and correlated default thresholds. The former results from dependence of firms on common macroeconomic factors, while the latter corresponds to direct inter-firm linkages. Having addressed this issuer interdependence, the predictions of our model are consistent with empirically well documented facts, in particular the clustering of defaults. We characterize joint conditional default probabilities as assessed by the imperfectly informed secondary market. The representation of dependence via (conditional) copulas is emphasized. We propose the default time copula as a consistent default correlation measure, which overcomes the limitations of existing covariance based measures. A case study is examined, where issuers' assets follow geometric Brownian motions and bond investors' threshold prior is uniform.
\end{abstract}

Key words: correlated defaults; default clustering; joint default distribution; copulas; incomplete information.

JEL Classification: G12; G13

\footnotetext{
*Address: Humboldt-Universität zu Berlin, SFB 373, Spandauer Strasse 1, D10178 Berlin, Germany, Phone +49 30 69503700, Fax +49 30 20935619, email giesecke@wiwi.hu-berlin.de I would like to thank Matthias Dörrzapf, Hans Föllmer, Ulrich Horst, Torsten Kleinow, Jean-Paul Laurent, and Franck Moraux for helpful discussions and suggestions. Financial support by Deutsche Forschungsgemeinschaft, Graduiertenkolleg Angewandte Mikroökonomik, is gratefully acknowledged.
} 


\section{Introduction}

In this paper we present a structural model of correlated multi-firm default, in which the default characteristics of issuers are not completely transparent to bond investors. The model predictions are consistent with several empirically documented facts.

A number of studies have investigated historical bond price and default data. They found, quite plausibly, that credit spreads as well as aggregate default rates are strongly related to general macro-economic factors such as the level of default-free interest rates, GDP growth rates, equity index returns and other business cycle indicators (see, for example, Duffee (1998) and Keenan (2000)). Another observation from the latest Moody's report is that there are default clusters around times of economic downturn. This clustering refers to infection effects and cascading defaults, where the default of a firm immediately increases the default likelihood of another firm dramatically. In its extreme form, a default directly triggers the default of another firm. Such effects can for instance be induced through mutual capital holdings, financial guarantees, or parent-subsidiary relationships. In a recession, default rates increase and so does the likelihood of observing infectious defaults. Recent evidence of the default clustering phenomenon includes the banking crisis in Japan.

These empirical observations have an important consequence: defaults of firms are stochastically dependent. We can distinguish two mechanisms leading to default dependence. First, the financial health of any firm depends on common factors related to the state of the general economy. Second, firms are also directly linked and thus the health of a particular firm also depends on the default status of other firms. A thorough understanding of these mechanisms and the resulting default dependence structure, going beyond simple linear default correlation measurement, is of vital importance for corporate security valuation, design and analysis of default insurance contracts, default risk aggregation and management, regulation of financial institutions, and the counteraction of financial crises. Direct inter-firm linkages deserve special emphasis because of their potentially dangerous effects.

The modeling of default risk and the valuation of default-prone securities on the level of an individual firm is now well understood (see Lando (1997) for a survey). But only relatively few approaches attempt to incorporate the interdependence of default events. In the class of intensity based models, where the stochastic structure of default is modeled through an intensity process, default correlation can be induced by correlation between intensities through time [Duffie \& Garleanu (2001), Jarrow, Lando \& Yu (2000)]. Such an approach leads to conditionally independent default events and would capture firms' dependence on common factors. To accommodate the fact that the default of some firm may directly increase the default likelihood of some closely associated firm, Jarrow \& Yu (2001) assume the intensity of a particular firm to be a 
function also of the default status of other firms. Similar ideas appear in Duffie \& Singleton (1998), Davis \& Lo (2001), and Schönbucher \& Schubert (2001). Lando (1998) and Lando (2000) discusses both dependence mechanisms in a credit ratings-based framework.

In the class of structural models, one takes as given the dynamics of a firm's asset value. A default takes place if assets are sufficiently low relative to liabilities. To model firms' dependence on common economic factors, one can assume that assets are correlated through time, cf. Kealhofer (1998), Gupton, Finger \& Bhatia (1997), and Zhou (2001). In this paper, we extend this approach in order to capture also the effects of direct firm linkages.

In our structural model a default is defined as the first time a firm's assets fall below some threshold value. In practice, however, it is typically difficult for bond investors to observe assets and threshold value. Duffie \& Lando (2001) recently examined term structures of credit spreads with imperfect asset information. Giesecke (2001) analyzes credit spreads in the general imperfect information case. It turns out that in the realistic incomplete information case the credit spreads implied by a structural model are empirically more plausible than with perfect observation. This is due to the fact that with incomplete observation the default is an unpredictable surprise event. In order to retain these desirable properties, in this paper we assume that bond investors cannot observe the threshold asset level at which a firm is liquidated. Bond investors form instead a prior distribution on firms' thresholds.

As with complete information, the joint dependence of firms on the state of the economy can be modeled through correlation between firms' assets. With incomplete information, there is an additional source for default correlation: Bond investors can account for direct linkages between firms by presuming dependence between the default thresholds of different firms. This dependence is reflected in investors' threshold prior distribution. The prior is updated with the default status information arriving over time. This procedure can be thought of as an re-assessment of a firm's financial health in light of the surprising default of some closely associated firm. Such an immediate re-assessment leads to jumps in credit spreads of surviving firms upon default events in the market. This is characteristic for the default clustering phenomenon and corresponds to the idea of contagion among events of defaults.

We characterize joint conditional default probabilities for any group of firms, given the incomplete information of the secondary market revealed over time. This default distribution is a crucial prerequisite for (multi-name) credit derivatives valuation and corporate bond portfolio risk measurement and management. We show that joint default probabilities are critically influenced by the dependence structures of both running minimum asset process and default thresholds. This confirms and complements recent results of Frey \& McNeil (2001). For the consistent measurement of default correlation we propose the copula of the default times. This copula overcomes the limitations of existing 
covariance based measures, since it separates the complete non-linear default dependence structure from marginal default behavior.

The representation of dependence between random quantities via copulas and the newly introduced conditional copulas is one of our methodological contributions. In addition, we recast the existing first-passage structural default models in a common framework by stressing the role of the running minimum asset process. This process tracks the historic low of the asset value.

The paper is organized as follows. In Section 2 we formulate the model. The joint conditional default time distribution is characterized in Section 3. In Section 4 we analyze the default dependence structure. In Section 5, we exemplify our general results in the special case where assets follow a geometric Brownian motion. Section 6 concludes. The Appendix contains the proofs.

\section{The Model}

\subsection{Firms, Defaults, and Information}

We consider an economy with a financial market. Uncertainty is modeled by a probability measure $P$. Investors are assumed to be risk-neutral; on the financial market they can trade in bonds issued by several firms. The index set of all firms is denoted $I=\{1,2, \ldots, n\}$, where $n<\infty$. We take as given some $\mathbb{R}^{n}$-valued stochastic process $V=\left(V^{1}, \ldots, V^{n}\right)$, and we denote by $\left(\mathcal{F}_{t}\right)_{t \geq 0}$ the filtration generated by $V$. The process $V^{i}=\left(V_{t}^{i}\right)_{t \geq 0}$ is Markovian, continuous, and normalized to satisfy $V_{0}^{i}=0 . V_{t}^{i}$ is a sufficient statistic for the expected discounted future cash flows of firm $i$ as seen from time $t$. We will therefore call $V^{i}$ asset process. The running minimum asset process $\left(M_{t}^{i}\right)_{t \geq 0}$ is defined by

$$
M_{t}^{i}=\min \left\{V_{s}^{i} \mid 0 \leq s \leq t\right\}
$$

A firm has issued non-callable consol bonds, paying coupons at some constant rate as long as the firm operates. When a firm stops servicing the coupon, we say it defaults. It then enters financial distress and some form of corporate reorganization takes place. Consistent with this time-homogeneous capital structure, bond investors suppose that there is a vector of default thresholds $D=\left(D_{1}, \ldots, D_{n}\right)$, such that firm $i$ defaults as soon as the asset value falls to the level $D_{i}<0$. The random default time is thus given by

$$
\tau_{i}=\min \left\{t>0 \mid V_{t}^{i} \leq D_{i}\right\}
$$

and we set $\tau=\left(\tau_{1}, \ldots, \tau_{n}\right)$. By convention, $V_{t}^{i}=V_{\tau_{i}}^{i}$ for $t \geq \tau_{i}$. With the running minimum asset process $M^{i}$ we obtain immediately

$$
\left\{\tau_{i} \leq t\right\}=\left\{M_{t}^{i} \leq D_{i}\right\}
$$


Bond investors are outside investors and their access to inside firm information is limited. While they observe firms' assets (through the stock market, for example) and publicly announced default incidents, we suppose that they cannot directly observe firms' default thresholds. That is, the filtration $\left(\mathcal{G}_{t}\right)_{t \geq 0}$ describing the information flow on the public bond market is defined by

$$
\mathcal{G}_{t}=\mathcal{F}_{t} \vee \sigma(\tau \wedge t)
$$

where $a \wedge b$ denotes the minimum of $a$ and $b$. In lack of threshold information, investors form a continuous and strictly increasing prior distribution $G$ on $D$, which we take as given. Thresholds are assumed to be independent of assets.

\subsection{Determinants of Correlated Default}

Default correlation - the stochastic dependence between defaults of different firms - has two distinctive sources in our model. First, all firms' health depends on common macro-economic factors, such as the stage of some business cycle, commodity prices, interest rates, or consumer behavior. This results in an indirect association of firms which we will call macro-correlation. We accommodate this macro-correlation by letting firms' asset values be correlated. Asset value dependence is formally described by the running minimum asset copula $C_{t_{1}, \ldots, t_{n}}^{M}$ of the vector $M_{t}=\left(M_{t_{1}}^{1}, \ldots, M_{t_{n}}^{n}\right)$. Assuming that the distribution function $H_{i}(\cdot, t)$ of $M_{t}^{i}$ is continuous on $(-\infty, 0]$ for fixed $t$, we can then represent the joint distribution of $M_{t}$ uniquely as

$$
P\left[M_{t_{1}}^{1} \leq x_{1}, \ldots, M_{t_{n}}^{n} \leq x_{n}\right]=C_{t_{1}, \ldots, t_{n}}^{M}\left(H_{1}\left(x_{1}, t_{1}\right), \ldots, H_{n}\left(x_{n}, t_{n}\right)\right),
$$

for all fixed $t_{i}>0$ and all $x_{i} \leq 0$. The copula $C_{t_{1}, \ldots, t_{n}}^{M}$ represents the complete dependence structure of the vector $M_{t} .{ }^{1}$ We refer to Nelsen (1999) for an introduction to copula functions.

Second, due to parent-subsidiary relationships or mutual capital holdings, firms are also directly linked. We will call this association on the microeconomic firm level micro-correlation. Bond investors account for this by presuming dependence between default thresholds of micro-correlated firms. This

\footnotetext{
${ }^{1}$ The distribution of $M_{t}$ is completely determined by that of the given asset process $V$. By virtue of (4), we can construct the running minimum asset copula as follows:

$$
C_{t_{1}, \ldots, t_{n}}^{M}\left(u_{1}, \ldots, u_{n}\right)=P\left[M_{t_{1}}^{1} \leq H_{1}^{-1}\left(u_{1}, t_{1}\right), \ldots, M_{t_{n}}^{n} \leq H_{n}^{-1}\left(u_{n}, t_{n}\right)\right],
$$

where $u \in[0,1]$ and $H_{i}^{-1}(\cdot, t)$ is the generalized inverse of $H_{i}(\cdot, t)$. If assets $V^{i}$ follow a Brownian motion, then both $H_{i}(\cdot, t)$ and the distribution function of $M_{t}$ in case $n=2$ are known, cf. Borodin \& Salminen (1996) and Rebholz (1994). Here the copula $C_{t, s}^{M}$ can be characterized more explicitly. We are not aware of results relating the copula of $V$ to that of $M$, which would provide a more direct way to construct $C_{t_{1}, \ldots, t_{n}}^{M}$.
} 
threshold dependence is formally described by the copula $C^{D}$ of the prior $G$, corresponding to the unique representation

$$
G\left(x_{1}, \ldots, x_{n}\right)=C^{D}\left(G^{1}\left(x_{1}\right), \ldots, G^{n}\left(x_{n}\right)\right), \quad x_{i} \leq 0,
$$

for marginal threshold distributions $G^{i}$, which can be thought of as the idiosyncratic prior component corresponding to firm-specific threshold determinants. We can think of the copula $C^{D}$ as representing industrial organization-related threshold determinants which are due to direct issuer linkages. To give an example, the default point of a firm being an subsidiary of another company will not only depend on its own firm-specific characteristics, but also on the type and extent of the linkage to the parent firm. The extent of the linkage may be related to the cost allocation between the entities and the dependence of the subsidiary on the human or capital resource inflow from the parent.

\section{Joint Distribution of Defaults}

The goal of this section is to characterize the joint distribution of defaults as assessed by the incompletely informed secondary market, given by

$$
F_{t}\left(T_{1}, \ldots, T_{n}\right)=P\left[\tau_{1} \leq T_{1}, \ldots, \tau_{n} \leq T_{n} \mid \mathcal{G}_{t}\right],
$$

and linking its properties to the stylized facts of historical default data. In Section 5, where we make specific assumptions on the evolution of assets and the threshold prior, we turn to a more explicit discussion of the general results derived in the following.

\subsection{General Case with Incomplete Information}

Investors start at time $t=0$ with the (given) prior threshold distribution $G$. With the passage of time, firms go bankrupt and thereby reveal information on their default threshold. Bond investors use that information to update their estimates on the thresholds of the remaining firms. In order to study this Bayesian updating procedure, let us define the process $\left(S_{t}\right)_{t \geq 0}$, taking values in the power set $\mathbb{P}(I)$ of the firm index set $I$, by

$$
S_{t}=\left\{i \in I: \tau_{i} \leq t\right\} \text {. }
$$

Since it collects the indices of all those bonds having defaulted by time $t$, we will call the set $S_{t}$ the default scenario at $t$. By (2) and the continuity of the asset process $V^{i}$, we get

$$
\left\{S_{t}=s\right\}=\bigcap_{i \in s}\left\{D_{i}=M_{\tau_{i}}^{i}\right\} \cap \bigcap_{i \in I-s}\left\{D_{i}<M_{t}^{i}\right\} .
$$


Given some default scenario $s \in \mathbb{P}(I)$ appears at time $t$, that means bond investors know that $D_{i}=M_{\tau_{i}}^{i}$ for all defaulted firms $i \in s$ and that $D_{i}<M_{t}^{i}$ for all operating firms $i \in I-s$. With any default scenario appearing at time $t$ we can hence associate a set $B\left(M_{t}, \cdot\right) \in \mathcal{B}_{-}^{n}$ such that

$$
\left\{D \in B\left(M_{t}, s\right)\right\}=\left\{S_{t}=s\right\} .
$$

In the following we relate the updated threshold belief to the prior.

Proposition 3.1. Let $L$ denote the joint law of $D$. On the set $\left\{S_{t}=s\right\}$, the a posteriori threshold belief is represented by the distribution

$$
P\left[D \in A \mid \mathcal{G}_{t}\right]=\frac{L\left(A \cap B\left(M_{t}, s\right)\right)}{L\left(B\left(M_{t}, s\right)\right)}, \quad A \in \mathcal{B}_{-}^{n} .
$$

As an example, let us consider a time $t$ such that $S_{t}=\emptyset$; i.e. there has been no default by $t$. Then the thresholds of all firms satisfy $D_{i}<M_{t}^{i}$ or, put another way, $D \in B\left(M_{t}, \emptyset\right)=\left(-\infty, M_{t}^{1}\right) \times \ldots \times\left(-\infty, M_{t}^{n}\right)$. Noting that $G\left(x_{1}, \ldots, x_{n}\right)=L\left(\left(-\infty, x_{1}\right) \times \ldots \times\left(-\infty, x_{n}\right)\right)$, Proposition 3.1 implies that the $\mathcal{G}_{t}$-conditional distribution function $G_{t}$ of the vector $D$ is given by

$$
G_{t}\left(x_{1}, \ldots, x_{n}\right)=\frac{G\left(x_{1} \wedge M_{t}^{1}, \ldots, x_{n} \wedge M_{t}^{n}\right)}{G\left(M_{t}^{1}, \ldots, M_{t}^{n}\right)}, \quad x_{i} \leq 0
$$

We now use the familiar idea of a copula to separate the dependence structure from the conditional threshold distribution. We thus introduce the process $\left(C_{t}^{D}\right)_{t \geq 0}$, where $C_{t}^{D}: \Omega \times[0,1]^{n} \times[0, \infty) \rightarrow[0,1]$ is the conditional threshold copula, representing the conditional threshold dependence structure:

$$
G_{t}\left(x_{1}, \ldots, x_{n}\right)=C_{t}^{D}\left(G_{t}^{1}\left(x_{1}\right), \ldots, G_{t}^{n}\left(x_{n}\right)\right), \quad x_{i} \leq 0,
$$

where $G_{t}^{i}$ is the marginal conditional threshold distribution. Let us denote by $I_{t}^{i}(u)=\inf \left\{x \geq 0: G_{t}^{i}(x) \geq u\right\}$ the generalized inverse of $G_{t}^{i}$. Since the $G_{t}^{i}$ are continuous for all fixed times $t<\tau_{i}$, the copula $C_{t}^{D}$ can be constructed via

$$
C_{t}^{D}\left(u_{1}, \ldots, u_{n}\right)=G_{t}\left(I_{t}^{1}\left(u_{1}\right), \ldots, I_{t}^{n}\left(u_{n}\right)\right),
$$

where $u_{i} \in[0,1]$ is $\mathcal{G}_{t}$-measurable. Let us observe that $C^{D}$ and $C_{t}^{D}$ are not equal in general; for an example we refer to Section 5 .

The (ordinary) copula couples marginal distributions with the joint distribution of some random vector. The copula linking the marginal survival functions with the joint survival function is called survival copula, cf. Nelsen (1999). Ordinary copula and survival copula express in an equivalent way the dependence structure of a given random vector. In analogy to $\left(C_{t}^{D}\right)_{t \geq 0}$, we introduce the conditional survival threshold copula process $\left(\bar{C}_{t}^{D}\right)_{t \geq 0}$. 
Proposition 3.2. The conditional survival threshold copula can be constructed from the conditional threshold copula via

$$
\bar{C}_{t}^{D}\left(u_{1}, \ldots, u_{n}\right)=\sum_{i_{1}=1}^{2} \cdots \sum_{i_{n}=1}^{2}(-1)^{i_{1}+\cdots+i_{n}} C_{t}^{D}\left(v_{1 i_{1}}, \ldots, v_{n i_{n}}\right),
$$

where $v_{j 1}=1-u_{j}$ and $v_{j 2}=1$ and $u_{i} \in[0,1]$ is $\mathcal{G}_{t}$-measurable.

We are now in a position to characterize the joint conditional default distribution (6), which describes both the individual default behavior of firms and the interrelation across the performance of firms. This distribution is the key for the solution of a variety of problems related to the measurement of dependent credit risks, including the aggregation of correlated default risks, and the analysis of derivative instruments having payoffs contingent on the performance of several reference entities.

Theorem 3.3 (Joint default distribution). The conditional joint default probability is for $T_{i}>t$ and $\max _{i} \tau_{i}>t$ given by

$$
F_{t}\left(T_{1}, \ldots, T_{n}\right)=E\left[\bar{C}_{t}^{D}\left(1-G_{t}^{1}\left(M_{T_{1}}^{1}\right), \ldots, 1-G_{t}^{n}\left(M_{T_{n}}^{n}\right)\right) \mid \mathcal{G}_{t}\right] .
$$

Let us consider a time $t$ such that $S_{t}=\emptyset$. From the Markov property of $V$ and the independence of $D$ and $V$, for all $x_{i} \leq M_{t}^{i}$ we have

$$
\begin{aligned}
P\left[M_{T_{1}}^{1} \leq x_{1}, \ldots, M_{T_{n}}^{n} \leq x_{n} \mid \mathcal{G}_{t}\right] & =P\left[M_{T_{1}}^{1} \leq x_{1}, \ldots, M_{T_{n}}^{n} \leq x_{n} \mid D<M_{t}, V_{t}\right] \\
& =P\left[M_{T_{1}-t}^{1} \leq x_{1}-V_{t}^{1}, \ldots, M_{T_{n}-t}^{n} \leq x_{n}-V_{t}^{n}\right] .
\end{aligned}
$$

Representing this probability with the running minimum asset copula, cf. (4), Theorem 3.3 implies that

$$
\begin{aligned}
F_{t}\left(T_{1}, \ldots, T_{n}\right)= & \int_{A} \bar{C}_{t}^{D}\left(1-G_{t}^{1}\left(x_{1}\right), \ldots, 1-G_{t}^{n}\left(x_{n}\right)\right) \\
& d C_{T_{1}-t, \ldots, T_{n}-t}^{M}\left(H_{1}\left(x_{1}-V_{t}^{1}, T_{1}-t\right), \ldots, H_{n}\left(x_{n}-V_{t}^{n}, T_{n}-t\right)\right),
\end{aligned}
$$

where we integrate over $A=\left(-\infty, M_{t}^{1}\right) \times \ldots \times\left(-\infty, M_{t}^{n}\right)$. This expression warrants further comment. First, with incomplete threshold observation there are two sources of default correlation: dependence of asset values (inducing the copula $C_{t_{1}, \ldots, t_{n}}^{M}$ ) and threshold dependence (inducing the copula $\bar{C}_{t}^{D}$ ). Second, joint default probabilities and hence the joint default risk profile of the secondary bond market critically depend upon the statistical properties of both running minimum asset copula $C_{t_{1}, \ldots, t_{n}}^{M}$ and conditional survival threshold copula $\bar{C}_{t}^{D}$. All else being equal, copulas $C_{t_{1}, \ldots, t_{n}}^{M}$ implying low values in all marginals simultaneously lead to an increased likelihood of joint defaults, corresponding to an extreme risk. This copula property is called lower tail dependence, 
cf. Nelsen (1999). $C_{t_{1}, \ldots, t_{n}}^{M}$ is determined by the initially chosen multivariate model of the asset processes $V^{1}, \ldots, V^{n}$, going beyond linear asset correlation. Likewise, copulas $\bar{C}_{t}^{D}$ implying high threshold values in all marginals simultaneously induce higher joint default probabilities. This corresponds to upper tail dependence. For a given time $t$, the copula $\bar{C}_{t}^{D}$ is determined by the chosen a priori threshold copula $C^{D}$. In Section 5 we examine the particular effects the properties of $C^{D}$ have on joint defaults.

Noting the definition of a copula, the default probability of a single firm is obtained from Theorem 3.3 by setting $n=1$. On $\left\{\tau_{i}>t\right\}$ we have

$$
F_{t}^{i}(T)=P\left[\tau_{i} \leq T \mid \mathcal{G}_{t}\right]=E\left[1-G_{t}^{i}\left(M_{T}^{i}\right) \mid \mathcal{G}_{t}\right], \quad t \leq T .
$$

If $D_{i}$ has a $\mathcal{G}_{t^{-}}$-conditional density $g_{t}^{i}$, we can equivalently write

$$
F_{t}^{i}(T)=\int_{-\infty}^{0} P\left[M_{T}^{i} \leq x \mid \mathcal{G}_{t}\right] g_{t}^{i}(x) d x, \quad t \leq T
$$

The mapping $t \rightarrow F_{t}^{i}(T)$ can for fixed $T$ be subject to jumps upon the default arrival of other firms $j \neq i$, given a sufficient degree of micro-correlation between the firms. To give an example, suppose that there are two firms $i$ and $j$ where each firm holds a substantial amount of the other's debt. If a firm defaults, the risk of the remaining firm to experience financial distress is increased substantially. Letting $\tau_{j}<\tau_{i}$, for a sufficiently large horizon $T$ the default probability $F_{t}^{i}(T)$ is likely to jump upwards at time $t=\tau_{j}$. The jump corresponds to an immediate re-assessment of firm $i$ 's future performance by bond investors, given the information that the directly associated firm $j$ has defaulted. Loosely, the stronger the link, the more intense the jump.

Bond prices implied by our model exhibit an analogous jump pattern. Consider a defaultable zero bond ${ }^{2}$ with maturity date $T$ and a recovery payment of $1-\delta_{i}$ at $T$. We let $\delta_{i} \in[0,1]$ be independent and $\mathcal{G}_{\tau_{i}}$-measurable, with expected value $\bar{\delta}_{i}$. Let $d(t, T)$ denote the time- $t$ price of a default-free zero bond maturing at $T$. Assuming that defaults are independent of riskless interest rates, the defaultable zero bond has at time $t<\tau_{i}$ a price of

$$
p_{i}(t, T)=d(t, T)-d(t, T) \bar{\delta}_{i} F_{t}^{i}(T), \quad t \leq T
$$

which is the value of a riskless zero bond less the expected default loss. The bond price of firm $i$ can jump upon default incidences of micro-correlated firms, which is consistent with the clustering of defaults and corresponds to the idea of contagion among events of defaults.

\footnotetext{
${ }^{2}$ In our model, the capital structure of the firms is based on consol bonds having no fixed maturity and paying out a constant coupon to the bond investors. We can strip the consol coupon into a continuum of zero coupon bonds with recovery being pro-rata based on the default-free market value that the strips contribute to the consol. As for the valuation of the consol bond, it is therefore enough to consider the valuation of the zero bonds.
} 
This effect is a consequence of two properties of our model. The first is that firms can be micro-correlated; macro-correlation does not play any role here. The second is that defaults are surprise events. ${ }^{3}$ Upon a default in the market, bond investors revise their a posteriori threshold belief $G_{t}$ immediately according to the degree of micro-correlation between the defaulted firm and the operating firms, cf. Proposition 3.1. This updating leads then to a revision of default probabilities via (9).

\subsection{Comparison: Perfect Information Case}

For comparison, let us briefly consider the situation when bond investors are perfectly informed and each $D_{i}$ is a known constant. Then, letting $t$ such that $S_{t}=\emptyset$, from Theorem 3.3 and the Markov property of $V$ we obtain

$$
\begin{aligned}
F_{t}\left(T_{1}, \ldots, T_{n}\right) & =P\left[M_{T_{1}-t}^{1} \leq D_{1}-V_{t}^{1}, \ldots, M_{T_{n}-t}^{n} \leq D_{n}-V_{t}^{n}\right] \\
& =C_{T_{1}-t, \ldots, T_{n}-t}^{M}\left(H_{1}\left(D_{1}-V_{t}^{1}, T_{1}-t\right), \ldots, H_{n}\left(D_{n}-V_{t}^{n}, T_{n}-t\right)\right) .
\end{aligned}
$$

With complete information, default dependence is induced by asset correlation only. Defaults are no surprise events any more and jumps in bond prices upon defaults of linked firms cannot appear. The approach of Zhou (2001), who models assets as correlated Brownian motions, shares these properties. For Brownian asset dynamics, the bivariate joint default probability $F_{t}\left(T_{1}, T_{2}\right)$ can be explicitly calculated using the results of Rebholz (1994) on the joint distribution of $\left(M_{T_{1}}^{1}, M_{T_{2}}^{2}\right)$. The above characterization offers in fact a general multivariate conditional representation of Zhou's (2001) formula in terms of the running minimum asset copula and marginal running minimum asset distribution. If we pass to the incomplete information situation, direct inter-firm linkages come into play, clustering effects can appear, and conditional default probabilities are provided by Theorem 3.3.

With complete information and for given $H_{i}\left(D_{i}, t\right)$, joint default probabilities depend on the statistical properties of the running minimum asset copula only. This is consistent with a result derived by Frey \& McNeil (2001) for general latent variable models based on complete information. For given marginal default probabilities, they showed that the copula of the latent variables completely determines the joint default probability of several issuers. In a simulation study, Frey \& McNeil (2001) demonstrate that the distribution of the total number of defaults is highly sensitive to the tail dependence properties of the latent variable copula. The latent variables can in our setup be interpreted as the assets' running minima.

\footnotetext{
${ }^{3}$ That is, each $\tau_{i}$ is a totally inaccessible or unpredictable $\left(\mathcal{G}_{t}\right)$-stopping time. That means that investors observe a default only in the moment where it occurs. For a detailed discussion of this property and its implications, see Giesecke (2001).
} 


\section{Characterizing Default Correlation}

The goal of this section is to clarify the structure of the association between firm defaults. There is a broad consensus in the existing literature to measure the pairwise default correlation between two firms $i$ and $j$ over some time period $[0, t]$ via the linear correlation coefficient

$$
\rho\left(N_{t}^{i}, N_{t}^{j}\right)=\operatorname{Cov}\left[N_{t}^{i}, N_{t}^{j}\right]\left(\operatorname{Var}\left[N_{t}^{i}\right] \operatorname{Var}\left[N_{t}^{j}\right]\right)^{-\frac{1}{2}},
$$

cf., for example, Zhou (2001), Hull \& White (2000), Kealhofer (1998), and Lucas (1995). $N_{t}^{k}=1_{\left\{t \geq \tau_{k}\right\}}$ is the default indicator for some default time $\tau_{k}$, which is not necessarily defined as in (1). Li (2000) considers the linear survival time correlation $\rho\left(\tau_{i}-t, \tau_{j}-t\right)$. In our model, $E\left[N_{t}^{i} N_{t}^{j}\right]$ and $E\left[N_{t}^{k}\right]$ are directly available from Theorem 3.3 and $\rho$ can be easily computed.

Measuring default correlation via $\rho\left(N_{t}^{i}, N_{t}^{j}\right)$ has however severe limitations. The problems arise from the fact that covariance is the natural measure of dependence only for joint elliptically distributed random variables [Embrechts, McNeil \& Straumann (2001)]. The vector $\left(N_{t}^{i}, N_{t}^{j}\right)$ has Bernoulli marginals with success probability $E\left[N_{t}^{k}\right]$; it is not joint elliptically distributed. Covariance is not the natural dependence measure anymore: it cannot be expressed as a function of the corresponding copula only. At least three problems with the interpretation of $\rho$ can then arise. First, besides the fact that $\rho$ measures linear dependence only, $\rho=0$ does not imply independence between the defaults of the two firms. Second, small correlations $\rho$ cannot be interpreted as implying weak dependence. Third, given the marginals $E\left[N_{t}^{k}\right]$, we have $-1<\rho<1$. In view of this, the conclusions drawn in Zhou (2001) and Lucas (1995) based on $\rho$ should be taken with care.

\subsection{Default Copula}

What is then a proper default dependence measure? A natural choice is the copula of the default indicator vector $\left(N_{t}^{1}, \ldots, N_{t}^{n}\right)$. Although not scalar-valued, this copula represents the complete default (event) dependence structure. But we face another problem here: since the marginal distributions of $\left(N_{t}^{1}, \ldots, N_{t}^{n}\right)$ are discrete, its copula is not unique anymore. ${ }^{4}$ To circumvent this problem, we propose the copula $C^{\tau}$ of the (continuous) default time vector $\tau$ as a measure of default correlation. The default time copula $C^{\tau}$ represents the complete default (time) dependence structure.

\footnotetext{
${ }^{4}$ Tajar, Denuit \& Lambert (2001) suggest a copula-type representation of bivariate random vectors with Bernoulli marginals. The representation is unique and carries all the information about the dependence structure.
} 
Theorem 4.1. Let $J^{i}$ denote the generalized inverse of $F_{0}^{i}$. The copula $C^{\tau}$ of the default times is for all $u_{i} \in[0,1]$ given by

$$
C^{\tau}\left(u_{1}, \ldots, u_{n}\right)=E\left[\bar{C}^{D}\left(1-G^{1}\left(M_{J^{1}\left(u_{1}\right)}^{1}\right), \ldots, 1-G^{n}\left(M_{J^{n}\left(u_{n}\right)}^{n}\right)\right)\right]
$$

As a joint distribution, $C^{\tau}$ satisfies a version of the Fréchet-bounds inequality $W^{-}(u) \leq C^{\tau} \leq W^{+}(u)$ for all $u \in[0,1]^{n}$, cf. Nelsen (1999). If $C^{\tau}=W^{-}$, then the defaults are perfectly negatively correlated (we speak of countermonotone defaults). If $C^{\tau}=W^{+}$, then the defaults are perfectly positively related (and we speak of comonotone defaults). If $C^{\tau}=\Pi$, where $\Pi\left(u_{1}, \ldots, u_{n}\right)=u_{1} \cdots u_{n}$, then the defaults are independent. This suggests a partial order on the set of (default) copulas, which can allow a comparison of default time vectors. Since a copula is a multivariate distribution function with standard uniform marginals, a pairwise copula is easily constructed by $C^{\tau}(1, \ldots, 1, u, 1, \ldots, 1, v, 1, \ldots, 1)$.

The copula $C^{\tau}$ captures the complete (non-linear) dependence across defaults. $C^{\tau}$ measures any correlation between the random variables $\tau_{1}, \ldots, \tau_{n}$, whether induced by macro or micro-correlation. The default dependence structure is therefore a function of both asset and threshold dependence structure, represented by $C^{M}$ and $C^{D}$. This is best seen when we rewrite Theorem 4.1:

$$
\begin{aligned}
C^{\tau}\left(u_{1}, \ldots, u_{n}\right)= & \int_{\mathbb{R}_{-}^{n}} \bar{C}^{D}\left(1-G^{1}\left(x_{1}\right), \ldots, 1-G^{n}\left(x_{n}\right)\right) \\
& d C_{J^{1}\left(u_{1}\right), \ldots, J^{n}\left(u_{n}\right)}^{M}\left(H_{1}\left(x_{1}, J^{1}\left(u_{1}\right)\right), \ldots, H_{n}\left(x_{n}, J^{n}\left(u_{n}\right)\right)\right) .
\end{aligned}
$$

From this it is also seen that, in line with intuition, the defaults are independent if and only if assets and thresholds are independent. Indeed, if $C^{D}=\Pi$, then $\bar{C}_{0}^{D}=\Pi$ by virtue of Proposition 3.2. Using the fact that $C_{t_{1}, \ldots, t_{n}}^{M}=\Pi$ for all $t_{i} \geq 0$, we have

$$
\begin{aligned}
C^{\tau}\left(u_{1}, \ldots, u_{n}\right) & =\int_{\mathbb{R}_{-}^{n}} \Pi_{i=1}^{n}\left(1-G^{i}\left(x_{i}\right)\right) d H_{1}\left(x_{1}, J^{1}\left(u_{1}\right)\right) \cdots d H_{n}\left(x_{n}, J^{n}\left(u_{n}\right)\right) \\
& =\prod_{i=1}^{n} E\left[1-G^{i}\left(M_{J^{i}\left(u_{i}\right)}^{i}\right)\right] \\
& =\prod_{i=1}^{n} u_{i} .
\end{aligned}
$$

From our discussion in Subsection 3.2, in a structural default model with observable thresholds the complete range of default dependence (from countermonotonicity through comonotonicity) can be induced by a suitable choice of the assets' running minima copula $C^{M}$. If thresholds are nonobservable and independent $\left(C^{D}=\Pi\right)$, it is easy to check that the range of achievable default time correlation is in fact limited: the $\tau_{i}$ are conditionally independent given the asset value paths. Under incomplete information, the full range of default dependence can only be achieved by admitting correlation between the 
thresholds. In Section 5, we show that even in the absence of asset correlation the complete range of default dependence can be induced through a suitable choice of the threshold copula $C^{D}$.

In various applications such as derivatives valuation it is often required to simulate correlated default times. On the basis of Theorem 4.1, it is possible to simulate a random vector $\left(\tau_{1}, \ldots, \tau_{n}\right)$ having a joint distribution given by Theorem 3.3 directly from the copula $C^{\tau}$, see Embrechts et al. (2001) for details on algorithms. This provides a theoretically consistent simulation approach, compared to an approach based on marginal default probabilities $F^{i}$ and a linear default correlation matrix.

\subsection{Rank Default Correlation}

A scalar-valued measure of dependence can perhaps provide more intuition about the degree of default correlation. In order to capture the complete dependence, we require such a measure to be defined on copula level. Rank correlation fulfills this requirement as we shall see. It measures the degree of monotonic dependence, whereas linear correlation measures the degree of linear dependence only. We can for example define Spearman's rank default correlation by

$$
\rho_{S}\left(\tau_{i}, \tau_{j}\right)=\rho\left(F_{0}^{i}\left(\tau_{i}\right), F_{0}^{j}\left(\tau_{j}\right)\right),
$$

where $\rho$ is the ordinary linear correlation and $F_{0}^{k}$ is the default probability of firm $k$. Since $\left(F_{0}^{i}\left(\tau_{i}\right), F_{0}^{i}\left(\tau_{i}\right)\right)$ has joint distribution $C^{\tau}$ (we have $F_{0}^{k}\left(\tau_{k}\right) \sim$ $U(0,1)), \rho_{S}$ is the linear correlation of the copula $C^{\tau}$. Kendall's rank correlation (cf. Section 5) can be used in an equivalent way to express the monotonic association among the default times. By using the definition of linear correlation, we obtain

$$
\rho_{S}\left(\tau_{i}, \tau_{j}\right)=12 \int_{0}^{1} \int_{0}^{1}\left(C^{\tau}(u, v)-u v\right) d u d v
$$

showing that Spearman's rank correlation depends on the copula only. Also, $\rho_{S}$ is seen to be a scaled version of the signed volume enclosed by the copula $C^{\tau}$ and the product copula $\Pi(u, v)=u v$. Thus $\rho_{S}$ is a measure of the average distance between the actual distribution of $\left(\tau_{i}, \tau_{j}\right)$ and their distribution given independence. Besides being invariant under (strictly) increasing transformations, $\rho_{S}$ has the following useful properties, which are easily verified using (14): $\rho_{S} \in[-1,1], \rho_{S}=1$ iff $C^{\tau}=W^{+}, \rho_{S}=-1$ iff $C^{\tau}=W^{-}$, and $\rho_{S}=0$ if $C^{\tau}=\Pi$. 


\section{A Specific Two-Firm Model}

The results in the previous sections have been established without specific assumptions on the law of the underlying asset processes and the prior threshold beliefs of the bond investors. In order to exemplify our findings, in this section we will examine a two-firm model based on geometric Brownian motion. In contrast to the existing approaches, the Brownian motions are chosen to be independent; default correlation is induced by default threshold dependence.

\subsection{Assets, Information, and Prior}

We consider a bond market where bonds of two firms, labeled 1 and 2, are traded. Let us suppose that the firms are positively related. For example, each firm could hold a substantial part of the other one's debt. Following the tradition in structural default modeling, we assume that the total market value $Z^{i}$ of firm $i$ follows a geometric Brownian motion:

$$
d Z_{t}^{i}=Z_{t}^{i}\left(m_{i} d t+\sigma_{i} d W_{t}^{i}\right),
$$

where $m_{i} \in \mathbb{R}$ and $\sigma_{i}>0$ are constant drift and volatility parameters, respectively. $\left(W^{1}, W^{2}\right)$ is a two-dimensional standard Brownian motion. This implies that the firm values itself are not correlated. This is plausible if the firms are from quite different industries, so that their earnings/cash flows and hence firm values are not directly related. Macro-correlation is hence not considered; we will focus on direct linkages between firm defaults.

(15) has unique solution $Z_{t}^{i}=Z_{0}^{i} e^{B_{t}^{i}}$, where $Z_{0}^{i}>0$ is the initial value of firm $i$ and $B^{i}$ is a Brownian motion with drift $\mu_{i}=m_{i}-\frac{1}{2} \sigma_{i}^{2}$, i.e.,

$$
B_{t}^{i}=\mu_{i} t+\sigma_{i} W_{t}^{i}
$$

The incomplete information available to the secondary market is modeled by the filtration $\left(\mathcal{G}_{t}\right)_{t \geq 0}$, which is generated by the firm value processes and the default indicator processes. In lack of complete information, investors form a prior on the default thresholds. The prior is separated into an idiosyncratic component and an interrelation component. We assume that the idiosyncratic prior is uniform. This corresponds to uninformed investors not having any specific knowledge on the barriers. Reflected by the same idiosyncratic prior for either firm, the secondary market believes that both firms have similar individual default characteristics. Since the firms' market value follows a strictly positive geometric Brownian motion process, the prior has support $\left(0, Z_{0}^{i}\right)$. Denoting the barrier by $\hat{D}_{i}$, the idiosyncratic prior is then represented by the distribution function $\hat{G}^{i}(x)=P\left[\hat{D}_{i} \leq x\right]=x / Z_{0}^{i}$. We set $\hat{D}=\left(\hat{D}_{1}, \hat{D}_{2}\right)$.

We assume that bondholders' interrelation threshold prior is modeled by the Clayton copula family

$$
C^{\hat{D}}(u, v ; \theta)=\left(u^{-\theta}+v^{-\theta}-1\right)^{-\frac{1}{\theta}}, \quad(u, v) \in[0,1]^{2}, \quad \theta>0 .
$$


The Clayton family belongs to the class of Archimedean copulas. For a complete account we refer to Lindskog (2000), who also shows that this family exhibits lower tail dependence. The parameter $\theta$ controls the degree of dependence between the random variables $\hat{D}_{1}$ and $\hat{D}_{2}$. We choose $\theta>0$, implying that $C^{\hat{D}}$ displays positive dependence. Specifically, $\lim _{\theta \rightarrow \infty} C^{\hat{D}}(u, v ; \theta)=$ $W^{+}(u, v)$, reflecting perfect positive dependence, and $\lim _{\theta \rightarrow 0} C^{\hat{D}}(u, v ; \theta)=$ $\Pi(u, v)$, corresponding to independence.

In order to assess the degree of dependence associated with $C^{\hat{D}}(u, v ; \theta)$ for a particular $\theta$, we can consider Kendall's rank correlation $\rho_{K}$, which measures the degree of monotonic dependence (see, for example, Nelsen (1999)). $\rho_{K}(\hat{D}) \in[-1,1]$ and the copula $C^{\hat{D}}$ are related via

$$
\rho_{K}(\hat{D})=4 \int_{0}^{1} \int_{0}^{1} C^{\hat{D}}(u, v ; \theta) d C^{\hat{D}}(u, v ; \theta)-1=\frac{\theta}{\theta+2},
$$

showing that $\rho_{K}$ is determined by the copula only. $\rho_{K}$ is thus invariant under strictly increasing transformations of the random variables.

From now on we set $V_{t}^{i}=B_{t}^{i}$ for $t \geq 0$ and $i=1,2$. That is, our 'asset process' $V^{i}$ in the sense of Section 2 is the log-firm value $B^{i}$. Notice that the running minimum asset copula satisfies $C_{t_{1}, t_{2}}^{M}=\Pi$ for all pairs $\left(t_{1}, t_{2}\right)$, due to the assets being independent. We thus introduce the threshold transformation $D_{i}=\ln \hat{D}_{i}-\ln Z_{0}^{i}$. Since $V_{0}^{i}=0$, the prior has now support $(-\infty, 0)$.

Proposition 5.1. The threshold prior on $D=\left(D_{1}, D_{2}\right)$ is represented by the unique joint distribution function

$$
G(x, y ; \theta)=\left(e^{-\theta x}+e^{-\theta y}-1\right)^{-1 / \theta}, \quad(x, y) \in \mathbb{R}_{-}^{2} .
$$

\subsection{Belief Updating}

Without loss of generality we set $\tau_{1}<\tau_{2}$, i.e., Firm 1 defaults before Firm 2. Taking into account Proposition 5.1, the conditional joint law of $D$ follows from Proposition 3.1 by straightforward calculations:

Corollary 5.2. Under the current assumptions, the a posteriori belief of the bond investors is represented by the conditional distribution

$$
G_{t}(x, y ; \theta)=\frac{1}{K_{t}(\theta)}\left(e^{-\theta\left(x \wedge M_{t}^{1}\right)}+e^{-\theta\left(y \wedge M_{t}^{2}\right)}-1\right)^{-1 / \theta} \quad \text { on } \quad\left\{S_{t}=\emptyset\right\},
$$

where $K_{t}(\theta)=G\left(M_{t}^{1}, M_{t}^{2} ; \theta\right)$ and, for $x \in\left[d_{1}=M_{\tau_{1}}^{1}, 0\right]$,

$$
G_{t}(x, y ; \theta)=\left(\frac{e^{-\theta d_{1}}+e^{-\theta\left(y \wedge M_{t}^{2}\right)}-1}{e^{-\theta d_{1}}+e^{-\theta M_{t}^{2}}-1}\right)^{-(1 / \theta+1)} \quad \text { on } \quad\left\{S_{t}=\{1\}\right\}
$$


Let us consider the conditional copula $C_{t}^{D}$ associated with the conditional distribution $G_{t}$, cf. (8). Using the inverse functions $I_{t}^{i}$ of the marginals $G_{t}^{i}$ derived in Corollary 5.2, for $t<\tau_{1}$ we can verify that

$$
C_{t}^{D}(u, v ; \theta)=G_{t}\left(I_{t}^{1}(u), I_{t}^{2}(v) ; \theta\right)=C^{D}(u, v ; \theta),
$$

so that the threshold copula is invariant under conditioning on $\left\{S_{t}=\emptyset\right\}$. We emphasize that this is not always the case in our setting. To give an example, suppose that the thresholds are comonotone, $C^{D}(u, v ; \theta)=u \wedge v$. Then on $\left\{S_{t}=\{1\}\right\}$, the copula $C_{t}^{D}$ must satisfy

$$
G_{t}(x, y ; \theta \rightarrow \infty)=1_{\left\{x \geq d_{1}, y \geq d_{1}\right\}}=C_{t}^{D}\left(1_{\left\{x \geq d_{1}\right\}}, 1_{\left\{y \geq d_{1}\right\}} ; \theta \rightarrow \infty\right),
$$

implying that $C_{t}^{D}(u, v ; \theta \rightarrow \infty)=u v$ for all $t \geq 0$.

\subsection{Default Distribution}

We now turn to the joint default distribution when both firms still operate. We will need the density $h_{i}(\cdot, t)$ of the running minimum $M_{t}^{i}$ of the Brownian motion $V_{t}^{i}$ with drift $\mu_{i}$ and volatility $\sigma_{i}$ (cf. Borodin \& Salminen (1996)):

$$
\begin{aligned}
h_{i}(x, t)= & \frac{1}{\sigma_{i} \sqrt{t}} \phi\left(\frac{-x+\mu_{i} t}{\sigma_{i} \sqrt{t}}\right) \\
& +\exp \left(\frac{2 \mu_{i} x}{\sigma_{i}^{2}}\right)\left[\frac{2 \mu_{i}}{\sigma_{i}^{2}} \Phi\left(\frac{x+\mu_{i} t}{\sigma_{i} \sqrt{t}}\right)+\frac{1}{\sigma_{i} \sqrt{t}} \phi\left(\frac{x+\mu_{i} t}{\sigma_{i} \sqrt{t}}\right)\right],
\end{aligned}
$$

where $\Phi$ (resp. $\phi$ ) is the standard normal distribution (resp. density) function.

Corollary 5.3. Under the current assumptions, the conditional joint distribution of the default times is for $t<T_{i}$ on the set $\left\{S_{t}=\emptyset\right\}$ given by

$$
\begin{aligned}
F_{t}\left(T_{1}, T_{2} ; \theta\right)= & \frac{1}{K_{t}(\theta)} \int_{-\infty}^{M_{t}^{2}} \int_{-\infty}^{M_{t}^{1}}\left(G(x, y)-G\left(x, M_{t}^{2}\right)-G\left(M_{t}^{1}, y\right)+1\right) \\
& \times h_{1}\left(x-V_{t}^{1}, T_{1}-t\right) h_{2}\left(y-V_{t}^{2}, T_{2}-t\right) d x d y .
\end{aligned}
$$

Figure 1 graphs the term structure of joint unconditional default probabilities $F_{0}(T, T ; \theta)$. The parameter for computations are as follows: $\mu_{1}=\mu_{2}=6 \%$ (the riskless rate), $\sigma_{1}=20 \%, \sigma_{2}=30 \%, M_{t}^{1}=-0.2$, and $M_{t}^{2}=-0.1$.

In Section 3 the effect of tail dependent threshold copulas $C^{D}$ on joint default probabilities has been discussed. We now quantify this effect by examining the shape of the distribution of the number of defaults. Specifically, we consider the variance $\operatorname{Var}\left[N_{T}\right]$ of the random variable $N_{T}=1_{\left\{\tau_{1} \leq T\right\}}+1_{\left\{\tau_{2} \leq T\right\}}$ for two different threshold copulas. The first is the Clayton copula which exhibits lower tail dependence. The second is the Gumbel copula

$$
C_{G u}(u, v ; \theta)=\exp \left(-\left[(-\ln u)^{\theta}+(-\ln v)^{\theta}\right]^{\frac{1}{\theta}}\right), \quad \theta \geq 1,
$$




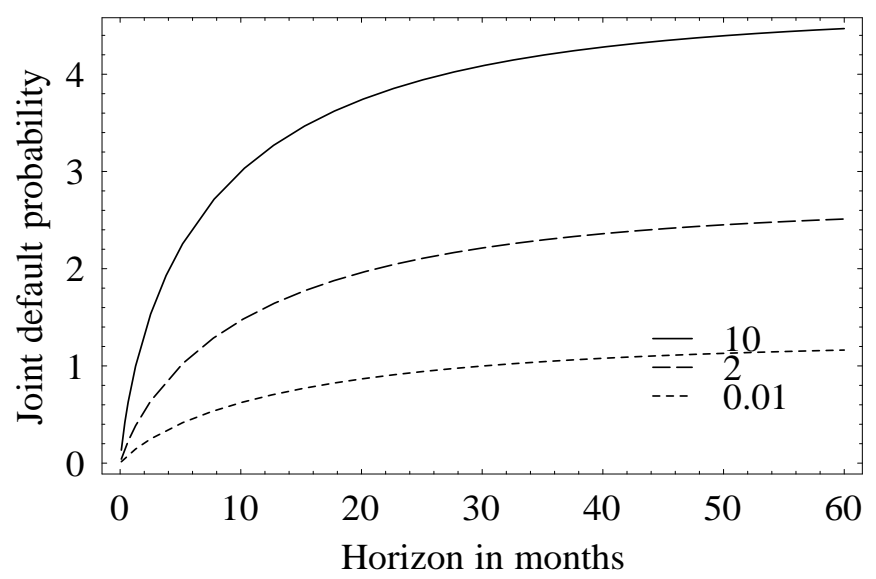

Figure 1: The term structure of joint unconditional default probabilities, varying $\theta$.

for $(u, v) \in[0,1]^{2}$, which exhibits upper tail dependence for $\theta>1$. We have $\lim _{\theta \rightarrow \infty} C_{G u}(u, v ; \theta)=W^{+}(u, v)$ and $C_{G u}(u, v ; 1)=\Pi(u, v)$. Kendall's rank correlation is given by $\rho^{K}=1-1 / \theta$, cf. Lindskog (2000). We now compare the loss variance $\operatorname{Var}_{G u}\left[N_{T}\right]$ that is obtained with the Gumbel copula (i.e. we set $\left.C^{\hat{D}}(u, v ; \theta)=C_{G u}(u, v ; \theta)\right)$ with the variance $\operatorname{Var}_{C l}\left[N_{T}\right]$ obtained with the Clayton copula in the original setup where $C^{\hat{D}}(u, v ; \theta)$ is given by (17). For a horizon of $T=12$ months, Figure 2 pictures the ratio $\operatorname{Var}_{G u}\left[N_{T}\right] / \operatorname{Var}_{C l}\left[N_{T}\right]$ as a function of the rank threshold correlation $\rho^{K}$. For any given positive degree of monotonic threshold dependence, $\operatorname{Var}_{G u}\left[N_{T}\right]$ exceeds $\operatorname{Var}_{C l}\left[N_{T}\right]$ by a factor of at most 2.3. Hence upper tail dependence of thresholds induces an extreme loss risk, as the default number distribution gets fat tails (benchmark is the lower tail dependence case (Clayton copula)). That is, the shape of the default number distribution differs significantly depending on the presumed threshold dependence structure $C^{\hat{D}}$, going beyond monotonic threshold dependence as measured by $\rho^{K}$. On the other hand, this also shows that for given (for example estimated) $\rho^{K}$ there remains a considerable amount of model risk in choosing the 'right' copula $C^{\hat{D}}$. This finding parallels that of Frey \& McNeil (2001), who study the effect of the asset copula on joint defaults: they find that the choice of the appropriate asset copula leaves the model risk.

From Corollary 5.3 we obtain directly the conditional default probability $F_{t}^{i}(T ; \theta)$ of firm $i$ for the horizon $T>t$. The term structure of Firm 2's default probabilities $F_{t}^{2}(T ; \theta)$ on the set $\left\{S_{t}=\{1\}\right\}$ is plotted in Figure 3. We observe that the stipulated degree of dependence has a significant effect on the default probability level. This level increases with longer horizons.

For $V_{t}^{2}=-0.05$, Figure 4 displays $F_{t}^{2}(T ; \theta)$ as a function of $\theta$ for various time horizons $T$ when both firms still operate. Somewhat surprising at a first 


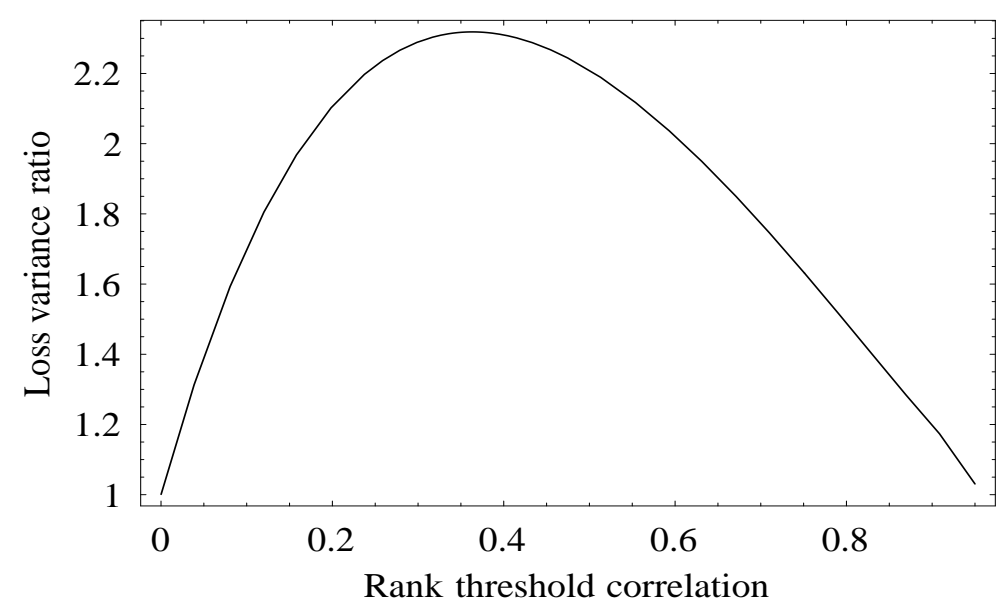

Figure 2: Default number variance ratio for Gumbel and Clayton threshold copulas as a function of rank threshold correlation.

glance, $F_{t}^{2}(T ; \theta)$ decreases in the degree of association $\theta$ (one can easily check that $G_{t}^{i}$ is increasing in $\theta$, cf. Corollary 5.2). This effect rests on the fact that investors observe the default status of all firms. The intuition here is that, given a positive degree of monotonic association between the firms, the information that Firm 1 has 'survived' up to time $t$ signals a 'good health' of Firm 2. The stronger the association, the more convincing is the fact that Firm 1 still operates, the higher bond investors rate Firm 2 and the lower $F_{t}^{2}(T ; \theta)$.

Figure 5 shows $F_{t}^{2}(T ; \theta)$ on $\left\{S_{t}=\{1\}\right\}$ as a function of $\theta$ for various time horizons $T$. We see that if Firm 1 has already defaulted, the default probability of Firm 2 is increasing in $\theta$ (again from Corollary 5.2, $G_{t}^{i}$ is decreasing in $\theta$ ). Here the intuition is as follows: under positive association between the firms, a default of Firm 1 lets investors conceive a bad state of Firm 2. The stronger the dependence, the lower is Firm 2's rating, and the higher its default probability for a given horizon. The effect of a given change of $\theta$ on $F_{t}^{2}(T ; \theta)$ is for all horizons higher if Firm 1 has defaulted, compared to the case where it still operates.

In Section 3, we have shown that default probabilities in our model can exhibit a distinguishing jump behavior: the default probability of a firm can for a fixed horizon exhibit jumps upon the default of correlated bonds. For a time horizon of $T=12$ months, Figure 6 displays this effect for Firm 2 . It shows $F_{t}^{2}(T ; \theta)$ for a fixed $t$ when both firms still operate and when Firm 1 has defaulted. The difference between the curves for a given $\theta$ is the jump that would $F_{t}^{2}(T ; \theta)$ experience if $t=\tau_{1}$. We observe that the jump size is increasing in the degree of association $\theta$ between the two firms. Clearly, if there is no relation between the firms, the default status of Firm 1 does not 


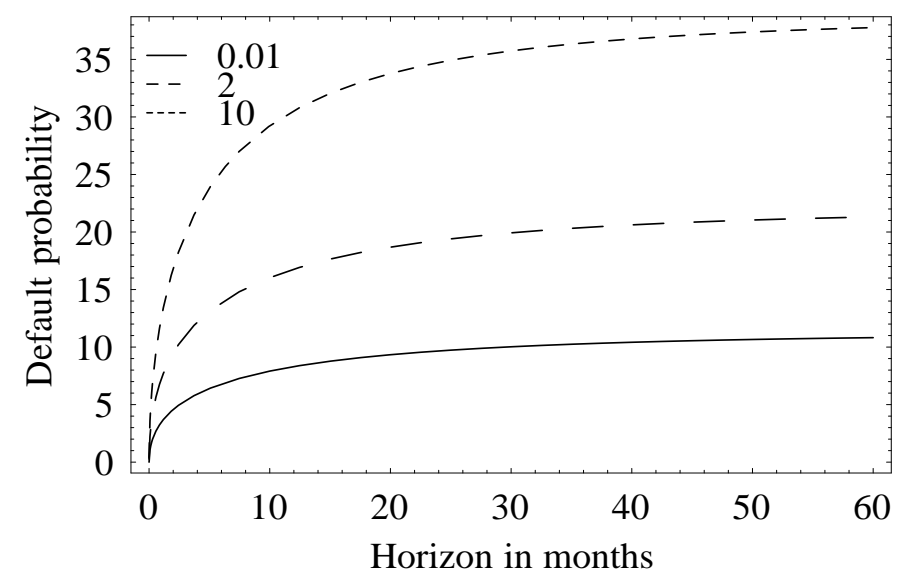

Figure 3: Term structure of conditional default probabilities for Firm 2, varying $\theta$ (Firm 1 has defaulted).

affect $F_{t}^{2}(T ; \theta)$. Note that the jump effect is critically dependent on the time horizon $T$. Fairly intuitive, with $T \rightarrow 0$ the jump effect vanishes and the jumps size is increasing in $T$.

The default correlation between the two firms can be measured by the copula $C^{\tau}$ of $\left(\tau_{1}, \tau_{2}\right)$, or Spearman's rank default time correlation $\rho_{S}\left(\tau_{1}, \tau_{2} ; \theta\right)$, defined in (13). Figure 7 plots $\rho_{S}$ as a function of the threshold association parameter $\theta$. We see that any desired degree of default correlation can be induced by a suitable choice of the Clayton-copula parameter $\theta$. Even with independent assets, the range of achievable correlation degrees is not restricted. It can be scaled from independence through comonotonicity.

\subsection{Estimating the Threshold Copula}

Let us briefly discuss two parametric methods to estimate the copula $C^{D}$. Yet another procedure is suggested by Jouanin, Rapuch, Riboulet \& Roncalli (2001), who use Moody's Diversity Score. The first method is based on the close relation between rank correlation $\rho_{K}(D)$ and copula $C^{D}$, and requires historical default threshold data. Using well-known methods (see, e.g., Gibbons (1988)), the empirical rank correlation $\rho_{K}$ can be estimated. Then the parameter $\theta$ of the copula family $C^{D}(\cdot ; \theta)$ can be fitted based on $(18)$.

The second procedure is based on historical bond price data, which is perhaps more readily available than default data. Let us consider firm $i$ 's zero bond prices $p_{i}^{\Pi}$ if the interdependence prior is $C^{D}=\Pi$, i.e. in the case where investors perceive the default thresholds of the firms to be independent. Corol- 


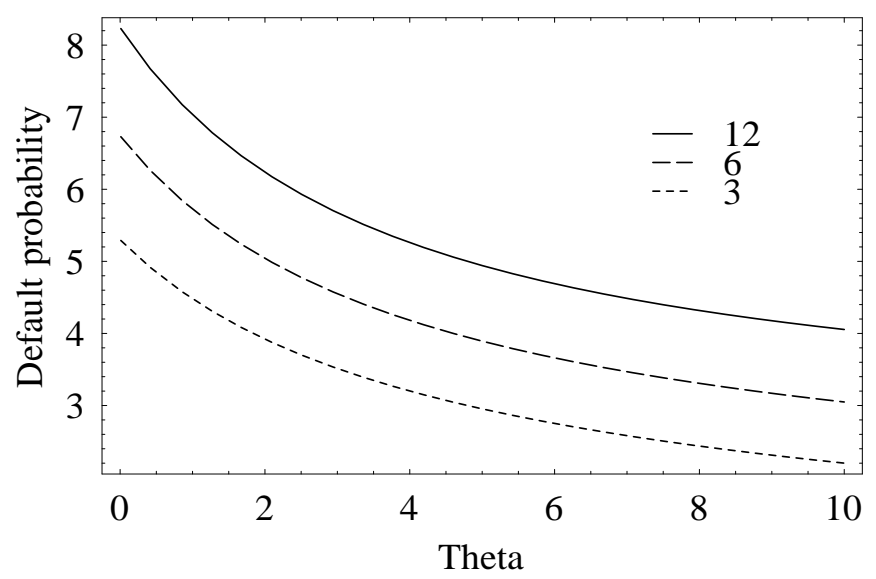

Figure 4: Conditional default probability Firm 2, varying horizon $T$ (both firms operate).

lary 5.2 yields

$$
G_{t}^{i}(x ; \theta \rightarrow 0)=\frac{G^{i}(x ; \theta \rightarrow 0)}{G^{i}\left(M_{t}^{i} ; \theta \rightarrow 0\right)}=e^{x-M_{t}^{i}}, \quad x \leq M_{t}^{i} .
$$

Since, from Corollary 5.3, default probabilities are given by

$$
F_{t}^{i}(T ; \theta)=1-\int_{-\infty}^{M_{t}^{i}} G_{t}^{i}(x ; \theta) h_{i}\left(x-V_{t}^{i}, T-t\right) d x
$$

we can now compute $p_{i}^{\Pi}(t, T)$ using (11). Assuming market efficiency and that corporate bond prices carry only a default risk premium, we can recover the implied secondary market's perception of the micro-dependence structure across firms by using $p_{i}^{\Pi}$ as a benchmark. For fixed $\left(t, T, \bar{\delta}_{i}\right)$, the difference $\Delta_{i}(t, T)=p_{i}(t, T)-p_{i}^{\Pi}(t, T)$ between the actually observed bond price of firm $i$ on the secondary market and $p_{i}^{\Pi}$ reflects the micro-correlation between the firms as assessed by the incompletely informed bond investors. If both firms still operate, then $F_{t}^{i}(T)$ is decreasing in $\theta$ (cf. Figure 4) and the bond price $p_{i}(t, T)$ is increasing in $\theta$. It follows that $\Delta_{i}(t, T) \geq 0$. Thus, if $\Delta_{i}(t, T)=0$ Firm $i$ is completely independent of Firm $j$. This provides a test for microindependence. If $\Delta_{i}(t, T)>0$, the parameter $\theta$ is positive and implicitly given by

$$
\frac{\Delta_{i}(t, T)}{\bar{\delta}_{i} d(t, T)}=\int_{-\infty}^{M_{t}^{i}}\left[e^{x-M_{t}^{i}}-\frac{\left(e^{-\theta x}+e^{-\theta M_{t}^{j}}-1\right)^{-1 / \theta}}{K_{t}(\theta)}\right] h_{i}\left(x-V_{t}^{i}, T-t\right) d x,
$$

where we have used (21) and Corollary 5.2. This suggests a method to calibrate the threshold copula $C^{D}$ from market data. 


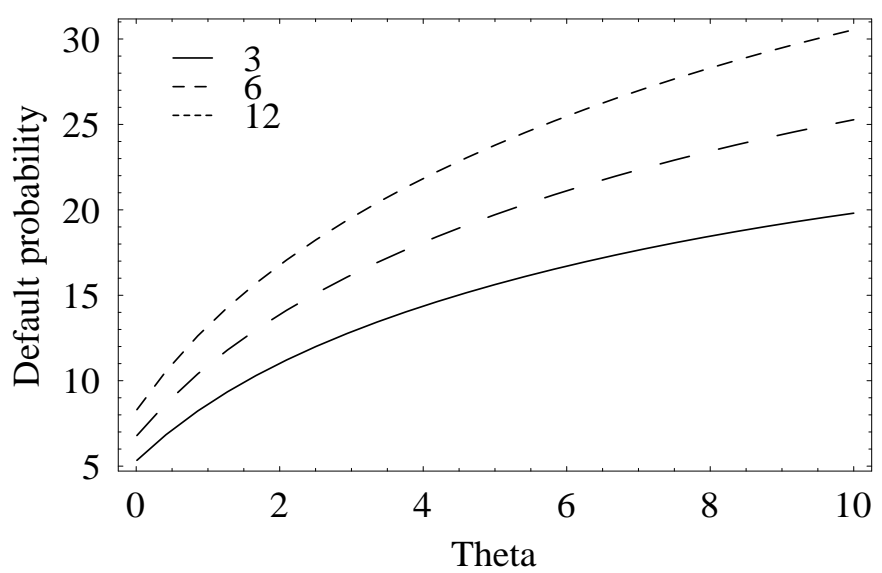

Figure 5: Conditional default probability Firm 2, varying horizon $T$ (Firm 1 has defaulted).

\section{Conclusion}

A thorough understanding of correlated multi-firm default mechanisms is of vital importance for corporate security valuation, design of default insuring contracts, risk measurement and management in financial institutions, and regulation of financial institutions. In this paper we proposed a structural model of correlated default, where bond investors are not informed about the asset threshold level at which the firm is liquidated. Default dependence was induced through asset value correlation and threshold level correlation. The latter corresponds to direct inter-firm linkages, which can lead to the often observed clustering of defaults. The predictions of our model are consistent with this clustering and other empirical regularities. Throughout, dependence has been represented by copula functions, providing new insights into the structure of firms' joint default behavior. For the consistent measurement of default correlation, we suggested the default time copula. 


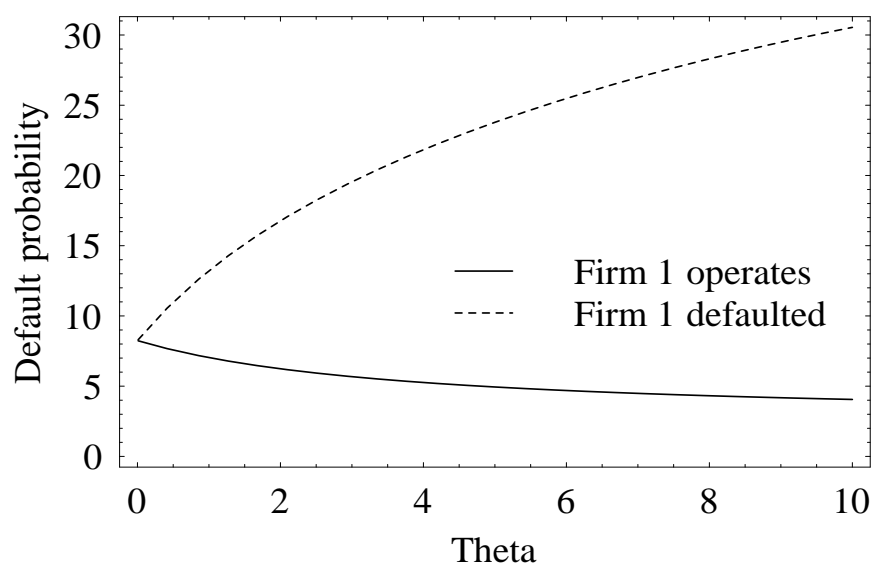

Figure 6: Conditional default probabilities of Firm 2 when both firms operate and when Firm 1 has defaulted.

\section{A Proofs}

Proof of Proposition 3.1. Since $M_{t} \in \mathcal{F}_{t} \subset \mathcal{G}_{t}$, from the structure of the $\sigma$-field $\sigma(\tau \wedge t)^{5}$ and from Bayes' Theorem, on the set $\left\{S_{t}=s\right\}$ we have

$$
\begin{aligned}
P\left[D \in A \mid \mathcal{G}_{t}\right] & =P\left[D \in A \mid \sigma(\tau \wedge t) \vee \mathcal{F}_{t}\right] \\
& =P\left[D \in A \mid D \in B\left(M_{t}, s\right), \mathcal{F}_{t}\right] \\
& =\frac{P\left[D \in A \cap B\left(M_{t}, s\right) \mid \mathcal{F}_{t}\right]}{P\left[D \in B\left(M_{t}, s\right) \mid \mathcal{F}_{t}\right]}
\end{aligned}
$$

This implies our assertion because $D$ is independent of $\mathcal{F}_{t}$.

Proof of Proposition 3.2. Fixing some $t \geq 0$, we have for $u_{i}=1-G_{t}^{i}\left(x_{i}\right) \in$ $[0,1]$ and any $x_{i} \leq 0$ the equalities

$$
\begin{aligned}
\bar{C}_{t}^{D}\left(1-G_{t}^{1}\left(x_{1}\right), \ldots, 1-G_{t}^{n}\left(x_{n}\right)\right) \\
=P\left[D_{1}>x_{1}, \ldots, D_{n}>x_{n} \mid \mathcal{G}_{t}\right] \\
=\sum_{i_{1}=1}^{2} \cdots \sum_{i_{n}=1}^{2}(-1)^{i_{1}+\cdots+i_{n}} P\left[D_{1} \leq v_{1 i_{1}}, \ldots, D_{n} \leq v_{n i_{n}} \mid \mathcal{G}_{t}\right] \\
=\sum_{i_{1}=1}^{2} \cdots \sum_{i_{n}=1}^{2}(-1)^{i_{1}+\cdots+i_{n}} C_{t}^{D}\left(G_{t}^{1}\left(v_{1 i_{1}}\right), \ldots, G_{t}^{n}\left(v_{n i_{n}}\right)\right)
\end{aligned}
$$

\footnotetext{
${ }^{5}$ The $\sigma$-field $\sigma(\tau \wedge t) \subseteq \mathcal{G}_{t}$ is generated by the events $\left\{\tau_{i} \leq u\right\}=\left\{M_{u}^{i} \leq D_{i}\right\}$ for $u \leq t$ and $i \in S_{t}$ as well as the atoms $\left\{\tau_{i}>t\right\}=\left\{M_{t}^{i}>D_{i}\right\}$ for $i \in I-S_{t}$.
} 


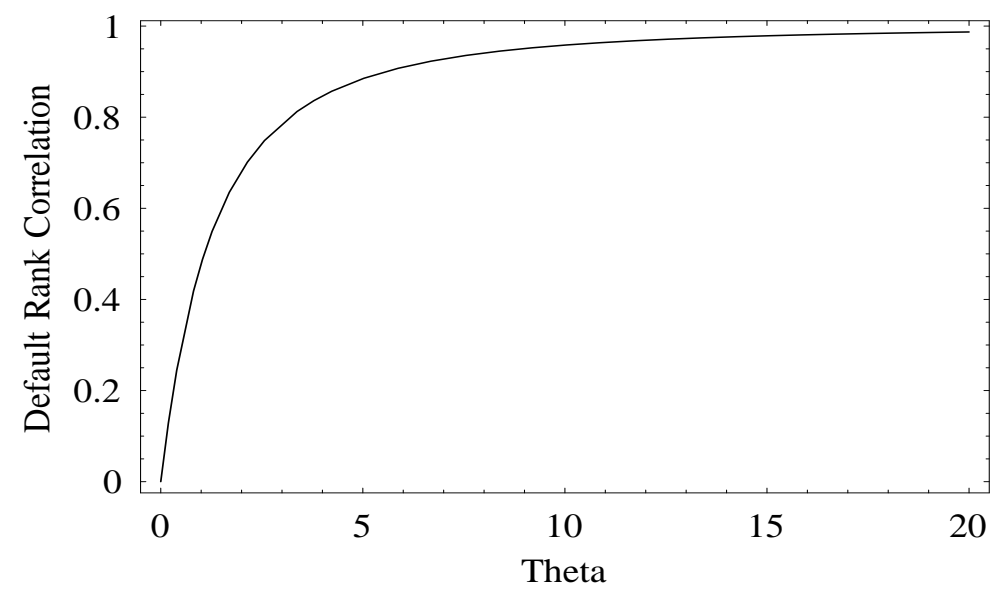

Figure 7: Spearman's rank default time correlation as a function of $\theta$.

where $v_{j 1}=x_{j}$ and $v_{j 2}=0$. The second equality can be verified using standard arguments. The third equality is a consequence of the key copula representation of joint distributions. Since $G_{t}^{i}(0)=1$ the claim follows.

Proof of Theorem 3.3. By (2), using the law of iterated expectations we can write

$$
\begin{aligned}
F_{t}\left(T_{1}, \ldots, T_{n}\right) & =P\left[D_{1} \geq M_{T_{1}}^{1}, \ldots, D_{n} \geq M_{T_{n}}^{n} \mid \mathcal{G}_{t}\right] \\
& =E\left[P\left[D_{1} \geq M_{T_{1}}^{1}, \ldots, D_{n} \geq M_{T_{n}}^{n} \mid \mathcal{G}_{t} \vee \mathcal{F}_{T}\right] \mid \mathcal{G}_{t}\right]
\end{aligned}
$$

where we set $T \geq \max _{i} T_{i}$. Since $D$ is independent of $\left(\mathcal{F}_{t}\right)_{t \geq 0}$ and $M_{T_{i}}^{i} \in \mathcal{F}_{T}$, our assertion now follows from the fact that the conditional survival threshold copula $\bar{C}_{t}^{D}$ satisfies

$$
\begin{aligned}
P\left[D_{1}>x_{1}, \ldots, D_{n}>x_{n} \mid \mathcal{G}_{t}\right] & =\bar{C}_{t}^{D}\left(P\left[D_{1}>x_{1} \mid \mathcal{G}_{t}\right], \ldots, P\left[D_{n}>x_{n} \mid \mathcal{G}_{t}\right]\right) \\
& =\bar{C}_{t}^{D}\left(1-G_{t}^{1}\left(x_{1}\right), \ldots, 1-G_{t}^{n}\left(x_{n}\right)\right)
\end{aligned}
$$

for any $x_{i} \leq 0$.

Proof of Theorem 4.1. Noting that $F_{0}^{i}(T)$ is continuous in $T$, the copula representation of the multivariate distribution function $F_{0}\left(T_{1}, \ldots, T_{n}\right)$ implies that

$$
C^{\tau}\left(u_{1}, \ldots, u_{n}\right)=F_{0}\left(J^{1}\left(u_{1}\right), \ldots, J^{n}\left(u_{n}\right)\right)
$$

The claim now follows directly from Theorem 3.3. 
Proof of Proposition 5.1. For fixed $Z^{i}>0, D_{i}$ is a strictly increasing transformation of $\hat{D}_{i}$. By the invariance property of copulas (cf. Nelsen (1999)), the copula $C^{D}$ of the transformed threshold vector $D$ remains unchanged:

$$
C^{D}(u, v ; \theta)=C^{\hat{D}}(u, v ; \theta) .
$$

It follows directly from the transformation $D_{i}=\ln \hat{D}_{i}-\ln Z_{0}^{i}$ that the idiosyncratic prior with respect to $D_{i}$ is represented by the marginal distribution function

$$
G^{i}(x)=P\left[D_{i} \leq x\right]=\hat{G}^{i}\left(Z_{0}^{i} e^{x}\right)=e^{x}, \quad x \leq 0,
$$

with density function $g^{i}(x)=e^{x}$. Since the $G^{i}$ are continuous, $G$ is now uniquely determined by $C^{D}$ and the $G^{i}$.

Proof of Corollary 5.3. The basis for the derivation in the Brownian case is Theorem 3.3 for $n=2$, which implies

$F_{t}\left(T_{1}, T_{2} ; \theta\right)=\int_{-\infty}^{M_{t}^{2}} \int_{-\infty}^{M_{t}^{1}} \bar{C}_{t}^{D}\left(1-G_{t}^{1}(x), 1-G_{t}^{2}(y)\right) d P\left[M_{T_{1}}^{1} \leq x, M_{T_{2}}^{2} \leq y \mid \mathcal{G}_{t}\right]$

Using the fact that $V^{1}$ and $V^{2}$ are independent, and hence $P\left[M_{T_{i}}^{i} \in d x \mid \mathcal{G}_{t}\right]=$ $P\left[M_{T_{i}}^{i} \in d x \mid V_{t}^{i}\right]=h_{i}\left(x-V_{t}^{i}, T_{i}-t\right) d x$, the default distribution can be written as

$$
\begin{aligned}
F_{t}\left(T_{1}, T_{2} ; \theta\right)= & \int_{-\infty}^{M_{t}^{2}} \int_{-\infty}^{M_{t}^{1}} \bar{C}_{t}^{D}\left(1-G_{t}^{1}(x), 1-G_{t}^{2}(y)\right) \\
& \times h_{1}\left(x-V_{t}^{1}, T_{1}-t\right) h_{2}\left(y-V_{t}^{2}, T_{2}-t\right) d x d y .
\end{aligned}
$$

The conditional survival threshold copula $\bar{C}_{t}^{D}$ is given by (8). In the bivariate case it simplifies to

$$
\bar{C}_{t}^{D}(u, v ; \theta)=C_{t}^{D}(1-u, 1-v ; \theta)+u+v-1 .
$$

From (19), $C_{t}^{D}=C^{D}$ and by using (17) we have that

$$
\bar{C}_{t}^{D}(u, v ; \theta)=\left((1-u)^{-\theta}+(1-v)^{-\theta}-1\right)^{-1 / \theta}+u+v-1, \quad \forall t \geq 0 .
$$

Substituting the marginals $G_{t}^{i}$, after simplification we get

$$
\bar{C}_{t}^{D}\left(1-G_{t}^{1}(x), 1-G_{t}^{2}(y)\right)=G_{t}(x, y)-G_{t}^{1}(x)-G_{t}^{2}(y)+1
$$

and the result follows from Corollary 5.2. 


\section{References}

Borodin, A. \& P. Salminen (1996), Handbook of Brownian Motion: Facts and Formulae, Birkhäuser, Basel.

Davis, Mark \& Violet Lo (2001), 'Infectious defaults', Quantitative Finance 1, 382-387.

Duffee, Gregory (1998), 'The relation between treasury yields and corporate yield spreads', Journal of Finance 53, 2225-2243.

Duffie, Darrell \& David Lando (2001), 'Term structures of credit spreads with incomplete accounting information', Econometrica 69(3), 633-664.

Duffie, Darrell \& Kenneth J. Singleton (1998), Simulating correlated defaults. Working Paper, GSB, Stanford University.

Duffie, Darrell \& Nicolae Garleanu (2001), 'Risk and valuation of collateralized debt obligations', Financial Analyst's Journal 57(1), 41-59.

Embrechts, Paul, Alexander J. McNeil \& Daniel Straumann (2001), Correlation and dependence in risk management, in M.Dempster, ed., 'Risk management: value at risk and beyond', Cambridge University Press, Cambridge.

Frey, Rüdiger \& Alexander J. McNeil (2001), Modeling dependent defaults. Working Paper, Department of Mathematics, ETH Zürich.

Gibbons, J. D. (1988), Non-Parametric Statistical Inference, Dekker, New York.

Giesecke, Kay (2001), Default compensator, incomplete information, and the term structure of credit spreads. Working Paper, Department of Economics, Humboldt-Universität zu Berlin.

Gupton, Greg, Christopher Finger \& Mickey Bhatia (1997), Creditmetricstechnical document. RiskMetrics Group, New York.

Hull, John \& Alan White (2000), 'Valuing credit default swaps ii: Modeling default correlations', Journal of Derivatives 8, 897-907.

Jarrow, Robert A., David Lando \& Fan Yu (2000), Default risk and diversification: Theory and applications. Working Paper, Cornell University.

Jarrow, Robert A. \& Fan Yu (2001), 'Counterparty risk and the pricing of defaultable securities', Journal of Finance 56(5), 555-576. 
Jouanin, J.-F., G. Rapuch, G. Riboulet \& T. Roncalli (2001), Modeling dependence for credit derivatives with copulas. Working Paper, GRO, Crédit Lyonnais.

Kealhofer, Stephen (1998), Portfolio management of default risk. Working Paper, KMV Corp.

Keenan, Sean (2000), Historical default rates of corporate bond issuers 19201999. Moody's Investors Services.

Lando, David (1997), Modeling bonds and derivatives with default risk, in M.Dempster \& S.Pliska, eds, 'Mathematics of Derivative Securities', Cambridge University Press.

Lando, David (1998), On rating transition analysis and correlation, in R.Jameson, ed., 'Credit Derivatives. Applications for Risk Management, Investment and Portfolio Optimization', Risk Publications, pp. 147-155.

Lando, David (2000), On correlated defaults in a rating-based model-common state variables versus simultaneous defaults. Working Paper, Department of Statistics and Operations Research, University of Copenhagen.

Li, David X. (2000), 'On default correlation: A copula function approach', Journal of Fixed Income 9, 43-54.

Lindskog, Filip (2000), Modeling dependence with copulas. Dissertation, Department of Mathematics, ETH Zürich.

Lucas, Douglas J. (1995), 'Default correlation and credit analysis', Journal of Fixed Income 11, 76-87.

Nelsen, Roger (1999), An Introduction to Copulas, Springer-Verlag, New York.

Rebholz, Joachim (1994), Planar diffusions with applications to mathematical finance. Ph.D. Dissertation, Department of Mathematics, University of California, Berkeley.

Schönbucher, Philipp \& Dirk Schubert (2001), Copula-dependent default risk in intensity models. Working Paper, Universität Bonn.

Tajar, Abdelouahid, Michel Denuit \& Philippe Lambert (2001), Copula-type representation for random couples with bernoulli marginals. Working Paper, Institute de Statistique, Université Catholique de Louvain.

Zhou, Chunsheng (2001), 'An analysis of default correlation and multiple defaults', Review of Financial Studies 14(2), 555-576. 